\title{
OUTCOME OF A LOW-CARBOHYDRATE DIET ON EGYPTIAN PATIENTS WITH NON ALCOHOLIC FATTY LIVER DISEASE
}

\author{
By \\ DOAA Z. ZAKY ${ }^{1 *}$ AND KHALID A. MANSOUR ${ }^{2}$ \\ Department of Tropical Medicine ${ }^{1 *}$ and Department of Internal Medicine ${ }^{2}$, \\ Faculty of Medicine, Ain Shams University, Cairo 11566, Egypt \\ ( ${ }^{*}$ Correspondence:drdoaazakaria@gmail.com) \\ Abstract
}

Non-alcoholic steatohepatitis (NASH) is a worldwide health problem and imposes a high cost on healthcare system, and NASH is associated with an unhealthy lifestyle.

This study investigated whether low carbohydrate diet intervention physical activity and affect intrahepatic fat content and hepatic enzymes.

The current interventional study included participants who were over 18 years with NASH $(\mathrm{n}=30)$ with BMI over $25 \mathrm{~kg} / \mathrm{m}^{2}$. The effects of proper nutritional therapy (moderate caloric restriction, low carbohydrate) was tested through personal dietary and physical assessment, parameters of the metabolic syndrome and insulin resistance in addition to liver biochemical and imaging study. The assessment was done at baseline and at 3 months by the same observer.

The results showed that after 3 months of intervention, patients had a significant decrease in caloric consumption, weight loss $(p<0.001)$ and HOMA-IR had significant improvements. Lipid profile after 3 months showed significant reduction including cholesterol and triglycerides levels $(p<0.05)$. Regarding liver profile significant decrease observed in aminotransferases levels; also there were significant improvements in AST/ALT ratio with decrease in severity of liver steatosis.

Keywords: Low carbohydrate diet, Non-alcoholic fatty liver disease, Hepatic fat content, Life style modification

\section{Introduction}

Non-alcoholic steatohepatitis (NASH) is an emerging health problem worldwide (Milic et al, 2015). It has common association with obesity and dietary habits (Eslamparast et al, 2017). NASH associated with high risk of development type 2diabetes, which are highly encountered in Egypt (ElTawdy et al, 2016. Non-alcoholic fatty liver disease (NAFLD) is a common cause of chronic liver disease, and its worldwide prevalence continues to increase with the growing obesity epidemic, diabetes and Hepatitis C (Vernon et al, 2011).

There is no effective drug yet for NASH, and weight reduction through diet modification is the cornerstone of treatment (Lee $e t$ $a l$, 2013). Carbohydrates play a major role in intrahepatic fat metabolism and developing insulin resistance (De Luis et al, 2010). Also, there was a marked correlation between carbohydrates intake percent and hepatic inflammation degree (Solga et al,
2004). The higher intake of carbohydrates and fatty acids especially saturated are associated with NASH (Gonzalez et al, 2013). By far no pharmacological agents proved to be effective in NASH management, the meta-analysis recommended physical activity and life style modification is a primary line in management NASH (Katagoni et al, 2017).

There was a growing interest in literature about advantages of low carbohydrate diet in NASH patients (Freedland and Aronson, 2009). Several report low-carbohydrate diet may be beneficial in improving fatty liver disease and associated obesity.

Although there are some reports regarding the non-significant difference between a low-carbohydrate or low fat diet on aminotransferase levels, (Rodriguez et al, 2011).

There is also an ongoing debate about its long-term safety in association with cardiovascular disease (Wang et al, 2016; Sumithran and Proietto, 2008). Insulin resistance 
is a main issue in NASH, low-carbohydrate may be an ideal choice in these types of patients (Scaglioni et al, 2013; Carvalhana et al, 2012)

From a clinical point of view, modifying lifestyle, increase period of physical activity and prescribing low carbohydrates diet offers a novel approach in reduction of intrahepatic fat content and emphasize the importance of integrating fitness assessment in management of NASH patients (Sullivan et al, 2012).

This study aimed to investigate the efficacy of a low carbohydrate diet with life style modification and physical activity on liver enzymes, lipid profiles and degree of liver steatosis among Egyptian population with NASH.

\section{Materials and Methods}

Study design, setting, and participants: This was conducted an interventional study with sample size 85 patients with NASH in Ain Shams University's Hospitals in the period from January 2018 to 2018 . The included cases with enlarged liver by US with sonographic criteria of hepatic steatosis and elevated liver enzymes as well as with the following criteria: 1- Adult patients > 18 years old, and 2- BMI over $25 \mathrm{~kg} / \mathrm{m}^{2}$. The excluded cases in the following conditions: 1- Patients with other etiology of chronic liver disease: hepatitis $\mathrm{C}$ hepatitis B, autoimmune liver disease, hemochromatosis, HIV infection, diabetics, 2- Patients with history of hepatotoxic or drug induced steatosis, alcoholic, and 3- Patients receiving anti-hyperlipdimic drugs.

Assessments of patients: Anthropometric measurements including: weight, height, BMI (body mass index), waist circumference WC. Based on the World Health Organization classification, overweight was defined as BMI between 25 and $29.9 \mathrm{~kg} / \mathrm{m}^{2}$, and obesity was defined as BMI over $30 \mathrm{~kg} / \mathrm{m}^{2}$. The measured waist circumference (in centimeters) by obtaining the distance of the mid-point between the $12^{\text {th }}$ ribs and iliac crest and hip circumference at the greater trochanters with the legs brought together. We measured arterial blood pressure and participant whose average blood pressure levels were greater or equal to $140 / 90$ $\mathrm{mmHg}$ or receiving antihypertensive medication was classified as hypertensive subjects.

Biochemical analysis:_Liver profile was done including: AST, ALT albumin, Metabolic profile included: Fasting serum lipids, total cholesterol, triglyceride, high-density lipoprotein and cholesterol.We set a value more than 2.0 on HOMA-IR index as the criteria of insulin resistance. The definition of the metabolic syndrome (MetS) was based on the IDF criteria (central obesity defined as waist circumference over $94 \mathrm{~cm}$ in men, over $80 \mathrm{~cm}$ in women or BMI over $30 \mathrm{~kg} / \mathrm{m}^{2}$ plus any two of the following factors: 1- Triglycerides (TG) level above 150 $\mathrm{mg} / \mathrm{dl}$, 2- High density lipoprotein-cholesterol (HDL-C) below $40 \mathrm{mg} / \mathrm{dl}$ in men or 50 $\mathrm{mg} / \mathrm{dl}$ in women, 3- Blood pressure above $130 / 85 \mathrm{mmHg}$ or on medication, and 4Fasting blood glucose $\geq 100 \mathrm{mg} / \mathrm{dl}$ or medication for diabetes.

Imaging: Hepatic steatosis (HS) was evaluated using ultrasonography criteria. Hepatic ultrasound is a sensitive procedure for detecting liver fat (sensitivity $91-100 \%$, specificity $93-100 \%$ ). We classified echogenicity as follows: a- Grade 0: normal echogenicity, b- Grade 1: slight, diffuse increase in fine echoes in liver parenchyma with normal view of diaphragm and hepatic vessel borders, c- Grade 2: moderate, diffuse increase in fine echoes with slightly impaired view of hepatic vessels and diaphragm, and d- Grade 3: marked increase in fine echoes with poor hepatic vessel borders, diaphragm, and posterior right lobe of the liver (Lowis and Mohanly, 2010).

Dietary program: Following approval was to join the study and completion of baseline assessments, enrolled patients received written individualized dietary plan which was developed for each participant according to his age, body weight and level of activity 
with all patients following maximum $50 \%$ or less of their total calories come from carbohydrates mainly complex carbs and low glycemic food items . All patients were advised to increase their physical activity during the study period and after. Reevaluation of the patients was done at the end of the three month of nutritional intervention

Nutritional intervention: A- Counseling was done individually every one to two weeks with final evaluation after 3 . All patients were required to submit a food diary at the baseline visit, as well as at 3 month, and each monthly visit. The food diary covers a 4 day period that includes 2 working days and 2 weekends. Foods were measured using standard measuring cups and spoons and weight for $100 \mathrm{~g}$; the input accuracy of the food diary was confirmed by using foodfrequency questionnaires.

No supplements were allowed in this period. Patients who missed more than $30 \%$ of the appointments or did not follow the dietary instruction for more than two weeks were considered noncompliant and were excluded from final analysis, B- Dietary plan : all patients were instructed to follow moderate restriction in calories, no more than 500-1000 caloric restriction than actual intake /day diet,with approximately less than $50 \%$ of daily caloric intake from carbohydrates , 30-35\% of total calories from fat (less than 7\% of total calories from saturated fat, less than $1 \%$ trans fatty acids, up to $20 \%$ of the total caloric intake from monounsaturated fatty acids, 5-10\% polyunsaturated fatty acids (PUFAs) proteins $20-25 \%$ of total calories (1.2 to $1.6 \mathrm{~g} / \mathrm{kg} / \mathrm{day}$ ), and $<5 \%$ of caloric intake from simple sugars occasionally mostly from fruits not on daily basis. They gave priority to low glycemic index food and nutrient that included vegetables and grains .Further recommendation included gradual increasing fibers intake to 25-30 g per day and low-calorie diet (500 kcal less than estimated energy needs), and C- Physical activity: A healthy lifestyle includes regular physical activity (PA). Regu- lar physical activity included 30 minutes of moderate intensity physical activities (e.g. brisk walking, jogging, cycling) for at least 5 days a week.

Statistical analysis: Data were statistically analyzed through statistical package of (SPSS) software version 13.0. Categorical data were expressed as number and percentage .Continuous data as in laboratory data were expressed as mean and SD. If data was normally distrusted we used Chi-square test $\left(x^{2}\right)$ (test of significance in categorical data). Paired $\mathrm{T}$ test was used for comparing between 2 dependent means. If data was not normally disturbed we used Mann Whitney $\mathrm{U}$ test for two-group compa-risons. The $\mathrm{P}$ value above 0.05 was con-sidered significant.

\section{Results}

Characteristics of population; The 85 patients were assessed for eligibility criteria. 30 subjects not meeting the inclusion criteria was excluded in addition to 10 subjects refused to join the study, 45 patients were enrolled of them 15 discontinued the intervention, final analysis of 30 patients was done after completion of 3 months so, the completion rate of this interventional study was $66.6 \%$. The average age of included patients was $38.95 \pm 7.1$ years .Overweight was present at baseline in $30 \%(n=9)$, obesity was present in $70 \%(\mathrm{n}=21)$ patients obesity was higher among females $72.2 \% v s .66 .6 \%$. The prevalence of Metabolic syndrome at baseline $46.6 \%(n=14)$, with higher prevalence among males $58.3 \%$ vs. $38.8 \%$.

Nutritional interventional, lifestyle and physical activity: At baseline, analysis of the 4-day by 24 four recall showed that caloric intake was in excess in almost all patients ,comparing to their actual needs (data not shown) the distribution of macronutrients was as follows, regarding proteins only $17.2 \pm 1.6 \%$,while their basal distribution of total carbohydrate and were within acceptable ranged $65.8 \pm 3.4 \% \& 23.9 \pm 3.5 \%$ respectively, regarding quantity but not quality, as dietary assessment revealed high percent- 
age of saturated fat ,trans fat and simple sugars at base line (data not given). Nutritional intervention caused significant reduction in carbohydrate \& total caloric intake, with increased physical activity; $30.7 \pm 19.3$ vs. $100.3 \pm 12.4$ / week, all $\mathrm{p}<0.001$ (Tab. 1).

After 3 month of individualized nutritional intervention depended mainly on carbohydrate restriction, highly significant loss weight $(p<0.001)$, with improvement in central obesity by highly significant decrease in WC of patients, the nutritional intervention resulted in categorizing patients into overweight class instead of obesity class at baseline assessment. Modification of weight management was associated with highly significant improvement in insulin resistance, diastolic blood pressure and overall metabolic syndrome $(\mathrm{p}<0.001)$, without significant sex difference for weight changes \& patients metabolic parameters (Tab. 2).

Nutritional intervention along 3 months caused significant improvement regarding AST, ALT $(p<0.05)$, albumin \& AST/ ALT ratio levels were not significantly changed. As to effect of intervention on hepatic steatosis, Nutritional interventional therapy was associated with reduction of both prevalence and severity of steatosis $(p<0.001)$. Regression of steatosis occurred even in absence of weight normalization in some patients (Tab. 3).

Table 1: Changes in life style including dietary modifications \& physical activity at baseline and 3 months

\begin{tabular}{|l|c|c|c|c|}
\hline \multicolumn{1}{|c|}{ Parameters } & Baseline & 3 months & P value & Significance \\
\hline Total caloric consumption in K calories & $2547.9 \pm 160.3$ & $1909.8 \pm 251.3$ & $<0.01$ & HS \\
\hline Protein $(\mathrm{g})$ & $70.2 \pm 11$ & $98.3 \pm 10.1$ & $<0.01$ & HS \\
\hline Carbohydrate $(\mathrm{g})$ & $288.2 \pm 29.4$ & $180.9 \pm 32.7$ & $<0.01$ & HS \\
\hline Lipid $(\mathrm{g})$ & $59.5 \pm 8.4$ & $60.3 \pm 11.3$ & 0.106 & $\mathrm{NS}$ \\
\hline physical activity(min/week) & $30.7 \pm 19.3$ & $100.3 \pm 12.4$ & $<0.01$ & HS \\
\hline
\end{tabular}

Table 2: Effect of nutritional intervention on metabolic parameters of patients at $0 \& 3$ months

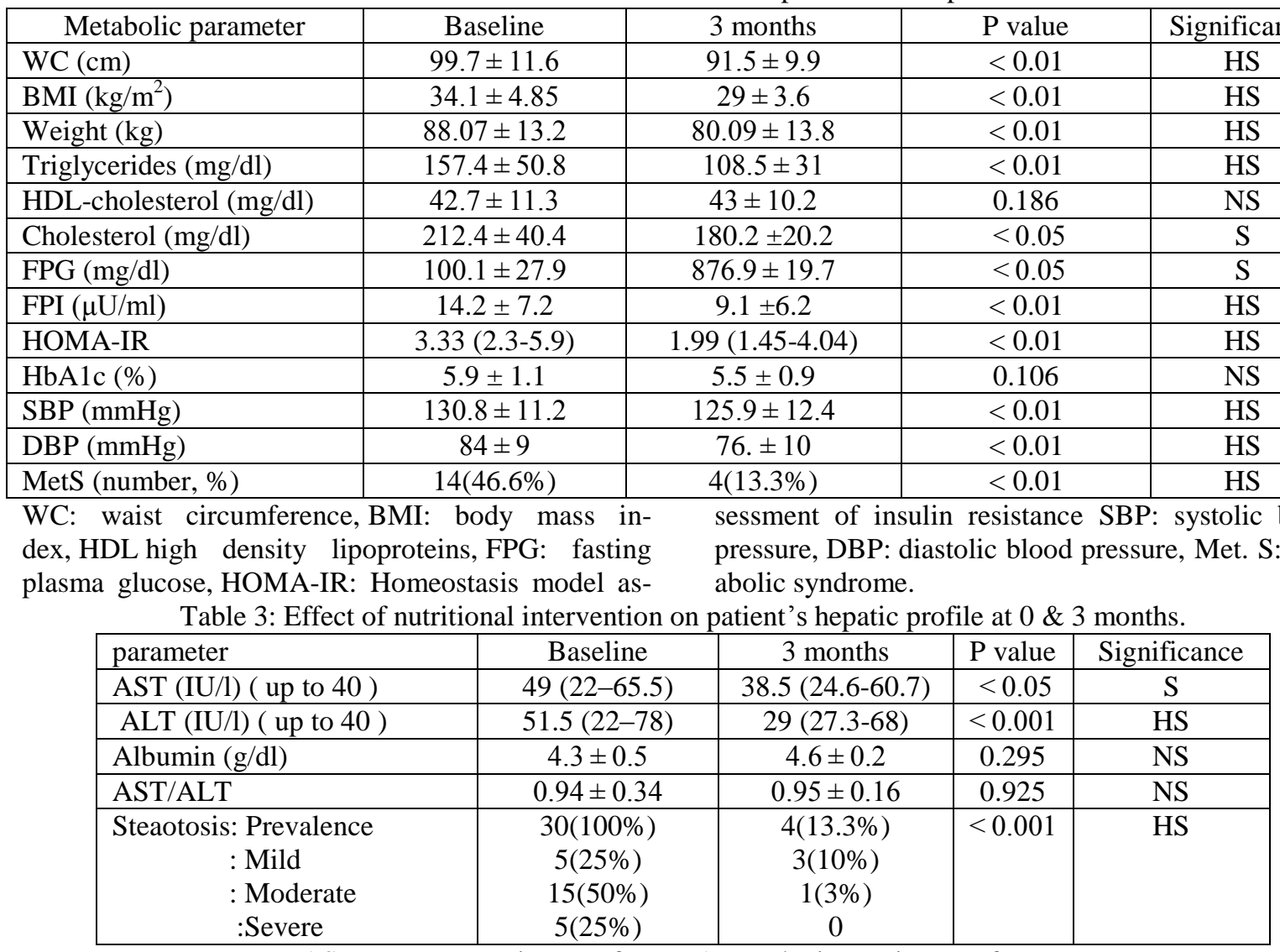

AST: aspartate aminotransferase, ALT: alanine aminotransferase, 


\section{Discussion}

In the present study, low carbohydrate diet program was effective in Egyptian NASH people. The present result showed low carbohydrate diet strategy was effective in reducing body weight but and improving metabolic parameters among Egyptian population.

Rafiei et al. (2018) reported that the main symptoms of the non-alcoholic fatty liver di sease (NAFLD) were occasionally, people with NASH or fibrosis (more advanced stages of NAFLD) might experience: a dull or aching pain in the top right of the tummy (over the lower right side of the ribs) extreme tiredness and unexplained weight loss.

Several studies have shown beneficial effects of dietary modification, weight loss and exercise in reducing insulin resistance and normalize level of aminotransferases in patients with NASH. Usually the first line of treatment recommended in NASH patient is weight reduction, physical activity and life style modification. However, the best optimal and effective approach in management of NASH was not clarified (Clark 2006; Park et al, 2010).

Similar trial conducted among Korean population to investigate effect of low-fat diet education with low-carbohydrate diet education in Korean NASH patients (Jang et $a l$, 2018). Unlike to Egyptian population, Asian population depends on rice as staple food and carbohydrate represent $60 \%$ of their energy intake (Jang et al, 2019). The low-carbohydrate diet program more efficient compared to low fat diet in decreasing intrahepatic fat content (Schwimmer et al, 2019).

Another trial conducted adolescent NASH population to compare the effect of regular diet versus to low sugar diet, after 8 weeks of follow up. Significant reduction in intrahepatic fat content was in group which consumed low sugar (Le et al, 2008).

The present study reached the same conclusion and showed that both low calorie diets could decrease liver enzymes similarly.
The effects of carbohydrates on the development of NASH differ depending on the carbohydrate type; degree of intrahepatic fact content is closely associated with certain type of carbohydrates especially ones with high glycemic food index (Muzio et al, 2007). Simple carbohydrates caused activation of hepatic lipogenesis and inhibit lipid oxidation leading to increase intra hepatic fat. This mechanism may be genetic related through activation of certain transcription factors. Animal study showed fat intake increase intrahepatic fat content but still data is deficient regarding humans. inflammatory factors are triggered by the carbohydrate and fat elements, which caused the metabolic disorders associated with the NASH (Le et al, 2008).

In clinical trials several issues were associated with low carbohydrate diet. First, still no clear evidence about the optimal amount of carbohydrate diet proportion .Variable definitions of low carbohydrate content found in literature, some define it by less than $40 \%$ and others defined it by less than $60 \%$. This may attributed to cultural differences in which macronutrient compositions vary widely. But no enough data whether the influence of carbohydrate composition was the same between different population (Pittas et al, 2005), as the high carbohydrate and fat consuming country (Galviz et al, 2015).

The effect of low carbohydrate on obesity was not clear in non-diabetic population compared to diabetic patient with insulin resistance which showed significant reduction in body weight (Chalasani et al, 2010). The strength of this study is the design as an intervention study. Compared to previous studies, the present diet program provides more detailed and accurate assessment for diet components and its effect on NASH population, degree of improvement of hepatic enzymes and its complication.

The present study was limited by relativity short period of follow up. A longer term trials was needed to carefully assess adverse effects of long-term alterations in the macro- 
nutrient composition.

\section{Conclusions}

Generally speaking, the association of the non-alcoholic fatty liver disease (NAFLD) with several other diseases has gained increased interest during the recent years.

The present study proved that the benefits of the individualized medical nutritional therapy with the low-carbohydrate diet, with physical activity in the management of the patients with NASH regarding improvement of body weight, lipid, insulin resistance and components of metabolic syndrome in addition to improvement in hepatic steatosis in terms of severity and prevalence.

\section{Acknowledgments}

The authors would like to thanks Dr. Reda Mokhtar, the Clinical Dietitian who kindly helped in the dietary assessment of the patients, the analysis of the 24 hours recalls and food frequency sheets and data collection.

\section{References}

Carvalhana, S, Machado, MV, Cortez-Pinto, H, 2012: Improving dietary patterns in patients with nonalcoholic fatty liver disease. Curr. Opin. Clin. Nutr. Metab. Care, 15, 5:468-73.

Chalasani, N, Guo, X, Loomba, R, Goodarzi, MO, Haritunians, T, et al, 2010: Genome-wide association study identifies variants associated with histologic features of nonalcoholic fatty liver disease. Gastroenterology 139:1567-76, 1576.e1-6.

Clark, JM, 2006: Weight loss as a treatment for nonalcoholic fatty liver disease. J. Clin. Gastroenterol. 40, 1:S39-43.

De Luis, DA, Aller, R, Izaola, O, Gonzalez, M, Conde, R, 2010: Effect of two different hypocaloric diets in transaminases and insulin resistance in nonalcoholic fatty liver disease and obese patients. Nutr. Hosp. 25, 5:730-5.

El-Tawdy, AHF, Ibrahim, EA, Abdallah, ES, Al Sakhawy EMA, Morsy, T.A, 2016: Screening for the diabetes mellitus: General information for patients to avoid foot amputation. EMMJ 71, 1:61 -74

Eslamparast, T, Tandon, P, Raman, M, 2017: Dietary composition independent of weight loss in the management of non-alcoholic fatty liver disease. Nutrients Jul 26;9, 8. pii: E800.

Freedland, SJ, Aronson, WJ, 2009: Words of wisdom. Re: Weight loss with a low-carbohydr- ate, Mediterranean, or low-fat diet. Dietary intervention randomized controlled trial (DIRECT) Group. N. Engl. J. Med. 359:229-41

Galaviz, KI, Narayan, K, Lobelo, F, Weber, MB, 2015: Lifestyle and the prevention of type 2 diabetes: A status report. Am. J. lifestyle Med. 12, 1:4-20.

Gonzalez, C, De Ledinghen, V, Vergniol, J, et al, 2013: Hepatic steatosis, carbohydrate intake, and food quotient in patients with NAFLD. Inter. J. Endocrinol. ID:428542, 4.

Harrison, SA, Day, CP, 2007: Benefits of lifestyle modification in NAFLD. Gut 56, 12:17609.

Jang, EC, Jun, DW, Lee, SM, Kyun, CY, Ahn, SB, 2018: Comparison of efficacy of lowcarbohydrate and low-fat diet education programs in non-alcoholic fatty liver disease: A randomized controlled study, Hepatol. Res. 48, 3: E22-9.

Jang, H, Kang, D, Chang, Y, Kim, Y, Lee, JS, et al, 2019: Non-alcoholic fatty liver disease and cerebral small vessel disease in Korean cognitively normal individuals. Sci. Rep. 2Oct 10; 9(1):14825. doi: 10.1038/s41598-019-51401-8.

Katsagoni, CN, Georgoulis, M, Papatheodoridis, GV, Panagiotakos, DB, Kontogianni MD, 2017: Effects of lifestyle interventions on clinical characteristics of patients with non-alcoholic fatty liver disease: A meta-analysis. Metabolism 68:119-32.

Lê, KA, Bortolotti, M, 2008: Role of dietary carbohydrates and macronutrients in the pathogenesis of nonalcoholic fatty liver disease. Curr. Opin. Clin. Nutr. Metab. Care 11, 4:477-82.

Lee, HL, Kim, KM, Kim, D, et al, 2013: Korean Association for the Study of the Liver (KASL), KASL clinical practice guidelines: Management of nonalcoholic fatty liver disease. Clin. Mol. Hepatol. 19, 4:325-9.

Lewis, JR, Mohanty, SR, 2010: Nonalcoholic fatty liver disease: a review and update. Dig. Dis. Sci. 55:560-78.

Milic, S, Mikolasevic, I, Krznaric, I, Stanic, M, Poropat, G, et al, 2015: Nonalcoholic steatohepatitis: Emerging targeted therapies to optimize treatment options. Drug Des. Devel. Ther. 9:4835-45.

Muzio, F, Mondazzi, L, Harris, WS, Sommariva, D, Branchi, A, 2007: Effects of moderate variations in the macronutrient content of the diet on cardiovascular disease risk factors in obese patients with the metabolic syndrome. Amer. 
J. Clin. Nutr. 86, 4:946-51.

Park, SH, Lee, KS, Park, HY, 2010: Dietary carbohydrate intake is associated with cardiovascular disease risk in Korean: Analysis of the third Korea National Health and Nutrition Examination Survey (KNHANES-III). Int. J. Cardiol. 139, 3:234-40.

Pittas, AG, Das, SK, Hajduk, CL, Golden, J, et al, 2005: A low-glycemic load diet facilitates greater weight loss in overweight adults with high insulin secretion but not in overweight adults with low insulin secretion in the CALERIE Trial. Diabetes Care 28, 12:2939-41.

Rodríguez-Hernández, H, Cervantes-Huerta, M, Rodríguez-Moran, M, Guerrero-Romero, F, 2011: Decrease of aminotransferase levels in obese women is related to body weight reduction, irrespective of type of diet. Ann. Hepatol. 10, 4:486-92.

Scaglioni, F, Marino, M, Ciccia, S, et al, 2013: Short-term multidisciplinary non-pharmacological intervention is effective in reducing liver fat content assessed non-invasively in patients with nonalcoholic fatty liver disease (NAFLD). Clin. Res. Hepatol. Gastroenterol. 37, 4:353-8.

Schwimmer, JB, Ugalde-Nicalo, P, Welsh, JA, Angeles, JE, et al, 2019: Effect of a low free sugar diet vs, usual diet on nonalcoholic fatty liver disease in adolescent boys: A randomized clinical trial. JAMA 321, 3:256-65.
Skilton, MR, Laville, M, Cust, AE, Moulin, P, et al, 2008: The association between dietary macronutrient intake and the prevalence of the metabolic syndrome. Br. J. Nutr. 100, 2:400-7.

Solga, S, Alkhuraishe, AR, Clark, JM, et al, 2004: Dietary composition and nonalcoholic fatty liver disease. Dig. Dis. Sci. 49, 10:1578-83 Sullivan, S, Kir, E, Mittendorfer, B, Patterson BW, Klein, S, 2012: Randomized trial of exercise effect on intrahepatic triglyceride content and lipid kinetics in nonalcoholic fatty liver disease. Hepatology 55, 6:1738-45.

Sumithran, P, Proietto, J, 2008: Ketogenic diets for weight loss: A review of their principles, safety and efficacy. Obes. Res. Clin. Pract. 2, 1:1-11.

Rafiei, R, Bemanian, M, Rafiei, F, Bahrami, M, Fooladi, L, et al, 2018: Liver disease symptoms in non-alcoholic fatty liver disease and small intestinal bacterial overgrowth. Rom J. Intern. Med. 56, 2:85-89

Vernon, GD, Baranova, AA, Younossi, ZM, 2011: Systematic review: The epidemiology and natural history of non-alcoholic fatty liver disease and non-alcoholic steatohepatitis in adults. Aliment. Pharmacol. Ther. 34, 3: 274-85

Wang, DD, Yanping, MD, Stephanie, E, Chiuve, et al, 2016: Association of specific dietary fats with total and cause-specific mortality. JAMA 176, 8:113-4. 